\title{
The Efficient Calculation of the Incomplete Beta-Function Ratio for Half-Integer Values of the Parameters $a, b$
}

\author{
By A. R. DiDonato and M. P. Jarnagin
}

1. Introduction. The incomplete beta function is defined as follows:

$$
B_{x}(a, b) \equiv \int_{0}^{x} t^{a-1}(1-t)^{b-1} d t
$$

where

$$
0 \leqq x \leqq 1, \quad a>0, \quad b>0 .
$$

When $x=1, B_{x}(a, b)$ is known as the (complete) beta function, and it can be expressed in terms of the gamma function by the well-known relation

$$
B_{1}(a, b)=\frac{\Gamma(a) \Gamma(b)}{\Gamma(a+b)},
$$

where the gamma function, with argument $s$, is defined by

$$
\Gamma(s) \equiv \int_{0}^{\infty} e^{-t} t^{s-1} d t, \quad s>0 .
$$

The ratio of (1) to (2) is called the incomplete beta-function ratio; it is represented by the symbol $I_{x}(a, b)$, thus,

$$
I_{x}(a, b) \equiv B_{x}(a, b) / B_{1}(a, b), \quad a>0, \quad b>0 .
$$

In probability theory, $I_{x}$ is often identified as a distribution function [2, p. 244] with mean $\mu$ and variance $\sigma^{2}$ given by

$$
\mu=a /(a+b), \quad \sigma^{2}=a b /\left[(a+b+1)(a+b)^{2}\right] .
$$

Throughout this paper the following restrictions are imposed on the parameters $a$ and $b$ :

$\{1\}$ They can assume only positive half-integer values, i.c., $a=k$ or $a=k-1 / 2$, $b=j$ or $b=j-1 / 2$, where $k$ and $j$ are positive integers.

$\{2\}$ They satisfy the inequalities

$$
1 / 2 \leqq a \leqq 10^{8}, \quad 1 / 2 \leqq b \leqq 60 .
$$

The purpose in this paper is to describe an efficient procedure, primarily for use on a high-speed digital computer, for the computation of $I_{x}(a, b)$ to high absolute accuracy, subject to constraints $\{1\}$ and $\{2\}$.

The function $I_{x}(a, b)$ is important in statistics because it can be directly related, or interpreted, in terms of basic probability functions such as the chi-square distribution, the $F$ (variance ratio) distribution, Student's $t$ distribution, and the dis-

Received November 15, 1966. Revised February 22, 1967. 
crete cumulative binomial distribution. It will be evident in the discussion given below that the restriction of $a$ and $b$ to half-integers is sufficient for expressing these probability distributions in terms of $I_{x}(a, b)$. Thus, $\{1\}$ is not as severe a limitation, from the standpoint of statistical applications, as might first be expected.

If $X_{1}^{2}, X_{2}^{2}$ are independent random variables which follow a chi-square distribution with $\nu_{1}, \nu_{2}$ degrees of freedom, respectively, then $X_{1}{ }^{2} /\left(X_{1}{ }^{2}+X_{2}{ }^{2}\right)$ follows a beta distribution where $a=\nu_{1} / 2, b=\nu_{2} / 2$. In [2, p. 243], it is shown that

$$
P\left\{X_{1}{ }^{2} /\left(X_{1}{ }^{2}+X_{2}{ }^{2}\right) \leqq x\right\}=I_{x}(a, b),
$$

where $P\{\alpha \leqq x\}$ is read as the probability that the random variable $\alpha$ is less than or equal to $x$.

The variance ratio or $F$ distribution with $\nu_{1}$ and $\nu_{2}$ degrees of freedom is related to $I_{x},[2$, p. 241-243], by

$$
P\left\{F \leqq F_{0}\right\}=1-I_{x}\left(\nu_{2} / 2, \nu_{1} / 2\right),
$$

where

$$
x=\nu_{2} /\left(\nu_{2}+\nu_{1} F_{0}\right), \quad F=\left(X_{1}{ }^{2} / \nu_{1}\right) /\left(X_{2}{ }^{2} / \nu_{2}\right) .
$$

The distribution of the ratio

$$
t=X /\left(X_{1}^{2} / \nu\right)^{1 / 2},
$$

where $X$ is a random variable following a normal distribution, and $X_{1}$ is a random variable following an independent chi-square distribution with $\nu$ degrees of freedom, gives rise to the Student's $t$ distribution, which can be expressed in terms of $I_{x},[2$, p. 238], as follows,

$$
P\left\{|t| \leqq t_{0}\right\}=1-I_{x}(\nu / 2,1 / 2),
$$

where

$$
x=\nu /\left(\nu+t_{0}^{2}\right) .
$$

In case $a$ and $b$ are integers, $I_{x}$ is directly related to the cumulative binomial distribution, [18, p. xvii], $E(n, r, x)$, as follows,

$$
E(n, r, x) \equiv \sum_{i=r}^{n} e(n, i, x)=I_{x}(r, n-r+1),
$$

where

$$
e(n, i, x) \equiv\left(\begin{array}{c}
n \\
i
\end{array}\right) x^{i}(1-x)^{n-i} .
$$

In spite of its importance, the number of available tables of $I_{x}$ is quite limited. The table of Karl Pearson [9] is the largest. For integer values of $a$ and $b$, there exist the cumulative probability tables of the Harvard Computation Laboratory [18] and the tables of the binomial probability distribution by the National Bureau of Standards [19]. A table of percentage points of $I_{x}(a, b)$, i.e., where $x$ is given as a function of $a, b$ and $I_{x}$, has been published by Thompson [12]. The requirements on the computing program, as stipulated above, greatly exceed the extent of any of these tabulations. 
A number of papers [4], [5], [7], [8], [10], [11], [14]-[17] have been published setting forth algorithms and/or programs for computing $I_{x}$. Most of these papers are discussed to some extent in [3]. None of these papers, however, is completely adequate for our purposes. Some of them are limited to analyses for establishing the asymptotic behavior of $I_{x}$ as $a$ or $b \rightarrow \infty$. The remaining publications cited above run afoul of at least one of the following major difficulties in computing $I_{x}$ :

\{a A straightforward binomial expansion of the integrand in (1) and a subsequent term-by-term integration results in an alternating series in powers of $x$ which cannot be used for large values of $a$. The eventual subtraction of consecutive terms of nearly equal absolute values causes a prohibitive loss in significant digits.

\{b $I_{x}$ is a function of three independent variables. Therefore, it is unlikely that one procedure or algorithm will suffice, and so it is necessary to devise a variety of schemes to encompass the ranges of $a, b$ which are contemplated.

\{c\} The extreme range of $a, 1 / 2 \leqq a \leqq 10^{8}$, introduces scaling problems in most procedures, because terms of the order of $\Gamma(a)$ occur in the intermediate calculations.

\{d\} The use of recurrence relations imposes the requirement of computing starting values, in which case one is confronted with the evaluation of $I_{x}(a, 1 / 2)$ for large $a$. This computation is not straightforward if efficiency and high accuracy are to be maintained.

\{e\} Closely connected to $\{\mathrm{d}\}$ is the fact that one must circumvent any procedure which requires a summation over $a$ elements, since this could entail the addition of $10^{8}$ terms. Such a procedure would destroy the efficiency of the program and very likely the accuracy as well.

2. An Efficient Method for Computing $I_{x}(a, b)$. This section contains the main results. Throughout the remainder of the paper $a$ and $b$ will be represented by $k$ or $k-1 / 2$ and $j$ or $j-1 / 2$, respectively. The upper bound on $j$ is rather arbitrary and may be increased, with a proportional increase in computing time. It will be assumed throughout that $I_{x}$ is to be computed to an accuracy of $\left\langle\log _{10} \epsilon\right\rangle$ decimal digits, where $\epsilon>0$ is assigned and

$$
\langle r\rangle \equiv \text { the greatest integer in } r .
$$

We will require the relationship

$$
I_{x}(a, b)=1-\bar{I}_{x}(a, b),
$$

where

$$
\bar{I}_{x}(a, b) \equiv I_{1-x}(b, a) .
$$

It is obtained from (1) by the substitution $u=1-t$.

The analysis upon which the computation of $I_{x}$ is based is separated into three cases A, B, C. The first two are somewhat straightforward; case C is not so. The characterization is as follows:

A: $a$ or $b$ is a positive integer not greater than 60 ,

$\mathrm{B}$ : neither $a$ nor $b$ is an integer, and $a<60$,

$\mathrm{C}: b$ is not an integer and $a>60$.

The basic equations for $\mathrm{A}$ and $\mathrm{B}$ are summarized below. The derivations as 
well as flow charts that specify the order in which the calculations are carried out and some other details which are not mentioned here are given in [3].

Case A. $b=j, 1 / 2 \leqq a \leqq 10^{8}$ and/or $a=k \leqq 60,1 / 2 \leqq b \leqq 60$; if $b=j$, $I_{x}$ can be computed from

$$
I_{x}(a, b)=\sum_{i=1}^{j} a_{i}
$$

where

$$
a_{i} \equiv[\Gamma(a+i-1) / \Gamma(a) \Gamma(i)] x^{a}(1-x)^{i-1} .
$$

If $a=k \leqq 60, I_{x}$ can be computed from the relation

$$
I_{x}(a, b)=1-\bar{I}_{x}(a, b)=1-\sum_{i=1}^{k} b_{i},
$$

where

$$
b_{i} \equiv[\Gamma(b+i-1) / \Gamma(b) \Gamma(i)] x^{i-1}(1-x)^{b} .
$$

The choice between (14) and (16) is made accordingly: if $b=j, a \neq k$, then use (14); if $a=k \leqq 60, b \neq j$, then use (16); if $a=k \leqq 60, b=j$, then use (14) if $j \leqq k$ or $(16)$ if $k<j$.

Every $a_{i}$ is positive and each is necessarily less than unity, since $I_{x} \leqq 1$; nevertheless, a scaling difficulty arises because the magnitude of the ratio of gamma functions, which appears in each $a_{i}$, can exceed the largest number a computer can handle. The same difficulty is manifest in the $b_{i}$ and in the $c_{i}$ given in (24) below. This difficulty is resolved as follows:

(1) the maximum $a_{i}$, say $a_{N}$, is determined by evaluating $N$ from

$$
N \equiv\left\{\begin{array}{cc}
\min \{\langle(a-1)(1-x) / x\rangle+1, j\} & \text { if } k \neq 1 \\
1 & \text { if } k=1
\end{array}\right\}
$$

This result is easily derived. One observes that, for $i<N, a_{i}<a_{i+1} ; a_{N+1} \leqq a_{N}$; for $i>N, a_{i+1}<a_{i}$.

(2) The logarithm of $a_{N}$ is then computed from

$$
\ln a_{N}=a \ln x+(N-1) \ln (1-x)+\ln \Gamma(a+N-1)-\ln \Gamma(a)-\ln \Gamma(N)
$$

from which $a_{N}$ is directly evaluated. In this way, the scaling difficulty for $a_{N}$ is resolved.

The remaining $a_{i}$ can now be computed from the following recurrence relations:

$$
\begin{aligned}
a_{i+1} & =\left[\frac{a+i-1}{i}\right](1-x) a_{i}, \quad N \leqq i \leqq j-1, \\
a_{i} & =\left[\frac{a+i-1}{i}\right]^{-1}(1-x)^{-1} a_{i+1}, \quad 1 \leqq i \leqq N-1,
\end{aligned}
$$

such that the $a_{i}$ with $i \geqq N$ are first computed and summed in increasing order of $i$ by (19), then the remaining $a_{i}$, starting at $i=N-1$, are computed and summed in decreasing order of $i$ by (20). 
Case B. $a=k-1 / 2<60, b=j-1 / 2$.

In this case, $I_{x}$ is computed from

$$
I_{x}(a, b)=I_{x}(a, 1 / 2)+\sum_{i=1}^{j-1}\left[\frac{\Gamma(a+i-1 / 2)}{\Gamma(a) \Gamma(i+1 / 2)}\right] x^{a}(1-x)^{i-1 / 2},
$$

where

$$
\begin{aligned}
I_{x}(a, 1 / 2) & =I_{x}\left(\frac{1}{2}, \frac{1}{2}\right)-[x(1-x)]^{1 / 2} \sum_{i=1}^{k-1}[\Gamma(i) / \Gamma(i+1 / 2) \Gamma(1 / 2)] x^{i-1} \\
I_{x}\left(\frac{1}{2}, \frac{1}{2}\right) & =(2 / \pi) \tan ^{-1}[x /(1-x)]^{1 / 2}, \quad 0 \leqq \tan ^{-1}[x /(1-x)]^{1 / 2} \leqq \pi / 2 .
\end{aligned}
$$

In terms of the notation

$$
\begin{aligned}
& c_{i} \equiv\left[\frac{\Gamma(a+i-1 / 2)}{\Gamma(a) \Gamma(i+1 / 2)}\right] x^{a}(1-x)^{i-1 / 2} \\
& d_{i} \equiv\left[\frac{\Gamma(i)}{\Gamma(i+1 / 2) \Gamma(1 / 2)}\right] x^{i-1}, \\
& I_{x}(a, b)=(2 / \pi) \tan ^{-1}[x /(1-x)]^{1 / 2}-[x(1-x)]^{1 / 2} \sum_{i=1}^{k-1} d_{i}+\sum_{i=1}^{j-1} c_{i} .
\end{aligned}
$$

The $d_{i}$ terms can obviously be computed recursively from

$$
d_{i+1}=x[2 i /(2 i+1)] d_{i}, \quad d_{1}=2 / \pi, \quad 1 \leqq i \leqq k-2,
$$

and the $c_{i}$ are obtained from

$$
\begin{aligned}
c_{i+1} & =[(a+i-1 / 2) /(i+1 / 2)](1-x) c_{i}, \quad N \leqq i \leqq j-2, \\
c_{i} & =[(i+1 / 2) /(a+i-1 / 2)](1-x)^{-1} c_{i+1}, \quad 1 \leqq i \leqq N-1 .
\end{aligned}
$$

The $c_{i}$ are summed in the same manner as the $a_{i}$, i.e., by first computing the maximum $c_{i}, c_{N}$, to resolve the scaling problem, and then summing in increasing order of $i$ from $N$ to $j-1$, followed by summing in decreasing order of $i$ from $N-1$ to 1 , where

$$
N=\min \{\langle[(a-1)(1-x) / x]+1 / 2\rangle, j-1\} .
$$

The above formulation for Cases $\mathrm{A}$ and $\mathrm{B}$ has the following advantages:

(1) all terms of each sum are of like sign,

(2) there are at most two main series to evaluate, with neither containing more than sixty terms,

(3) the magnitude of the factors appearing in $a_{i}$ and $c_{i}$ are kept under control without loss of accuracy,

(4) the computation of the successive $a_{i}, b_{i}, c_{i}$ is efficient.

Case C. $a>60, b=j-1 / 2$.

A somewhat more detailed discussion of this case will be given, since it is the most difficult one. The beta ratio is again given by (21); however, (22) cannot be used to evaluate $I_{x}(a, 1 / 2)$ because the summation runs to $k-1$. Thus the problem here reduces to finding an efficient method for computing $I_{x}(a, 1 / 2)$ when $a>60$.

The method chosen to evaluate $I_{x}(a, 1 / 2)$ in this case was that of Gaussian quadrature [6, p. 319]. This procedure was chosen because the truncation error, $E$ $[6$, p. 324], could be sharply and rigorously bounded, and, moreover, the derived 
error bound, $E^{\prime}$, showed that an amazingly low order could be used to obtain the desired accuracy. For example, the Gaussian quadrature of order ten would suffice for $10 \mathrm{D}$ accuracy in $I_{x}(a, 1 / 2)$.

Lack of space prohibits giving the complete details of the proof for bounding $E$; they are, however, given in [3]. The basic steps in the proof are outlined after some preliminary notations and inequalities are established.

The transformation $t=1-u^{2}$ applied to $B_{x}(a, 1 / 2)$ gives

$$
B_{x}(a, 1 / 2)=2 \int_{(1-x)^{1 / 2}}^{1}\left(1-u^{2}\right)^{a-1} d u .
$$

The symbols $M, \lambda$ are introduced through the following definitions:

$$
\begin{gathered}
M \equiv B_{1}(a, 1 / 2)=[\Gamma(a) \Gamma(1 / 2) / \Gamma(a+1 / 2)] \sim(\pi / a)^{1 / 2}, \quad(a \rightarrow \infty), \\
I_{x}(a, 1 / 2 ; \lambda) \equiv \frac{2}{M} \int_{(1-x)^{1 / 2}}^{\lambda}\left(1-u^{2}\right)^{a-1} d u
\end{gathered}
$$

In this notation, we have

$$
I_{x}(a, 1 / 2)=I_{x}(a, 1 / 2 ; \lambda)+I_{1-\lambda^{2}}(a, 1 / 2),
$$

where the last term is proportional to the area under the curve $\left(1-u^{2}\right)^{a-1}$ from $u=\lambda$ to $u=1$. The purpose of this representation is to choose $\lambda$ such that $I_{1-\lambda^{2}}(a, 1 / 2)<\epsilon^{\prime \prime}$, where $\epsilon^{\prime \prime}$ is another preassigned small number.

From (33), after writing the integrand as an infinite product of exponentials,

$$
\begin{aligned}
I_{1-\lambda^{2}}(a, 1 / 2 ; 1) \leqq & \frac{2}{M} \exp \left[(a-1)\left(-\sum_{2}^{\infty} \lambda^{2 i} / i\right)\right] \int_{\lambda}^{1} \exp \left[-(a-1) u^{2}\right] d u \\
= & \frac{2}{M} \frac{1}{(a-1)^{1 / 2}} \exp \left[(a-1)\left(-\sum_{2}^{\infty} \lambda^{2 i} / i\right)\right] \\
& \times\left[\int_{\lambda(a-1) 1 / 2}^{\infty} \exp \left[-z^{2}\right] d z-\int_{(a-1) 1 / 2}^{\infty} \exp \left[-z^{2}\right] d z\right],
\end{aligned}
$$

where $z=(a-1)^{1 / 2} u$. However, the last integral in (35) is negligible for $a \geqq 60$, since

$$
\int_{x}^{\infty} \exp \left[-z^{2}\right] d z \leqq(\sqrt{ } \pi / 2) \exp \left[-x^{2}\right], \quad[1, \text { p. } 298] .
$$

Another application of this inequality to (35), after the last integral has been dropped, yields

$$
\begin{aligned}
I_{1-\lambda^{2}}(a, 1 / 2 ; 1) & \leqq \frac{\sqrt{ } \pi}{M(a-1)^{1 / 2}} \exp \left[(a-1)\left(-\sum_{1}^{\infty} \lambda^{2 i} / i\right)\right] \\
& =\frac{\sqrt{ } \pi}{M(a-1)^{1 / 2}}\left(1-\lambda^{2}\right)^{a-1} \leqq \epsilon^{\prime \prime} .
\end{aligned}
$$

The smallest value of $\lambda$ that satisfies (36) gives $\lambda\left(\epsilon^{\prime \prime}\right)$, namely,

$$
\lambda\left(\epsilon^{\prime \prime}\right)=\left[1-\left(M \epsilon^{\prime \prime}((a-1) / \pi)^{1 / 2}\right)^{1 /(a-1)}\right]^{1 / 2} \sim\left[-\frac{1}{a-1} \ln \epsilon^{\prime \prime}\right]^{1 / 2},
$$

$$
(a \rightarrow \infty) \text {. }
$$


One can now deduce from (36) and (37) that the upper limit of integration (unity) in (31) can be replaced by $\lambda\left(\epsilon^{\prime \prime}\right)=\lambda$. Moreover, if $\lambda<(1-x)^{1 / 2}$ then the value of $I_{x}(a, 1 / 2)$ is less than $\epsilon^{\prime \prime}$, a fact that is easily concluded from (34) and (36).

These introductory results will be useful in obtaining the bound $E^{\prime}$ (given by (45)) on $E$. The exact error term, $E$, associated with the use of Gaussian quadrature of order $m[6, \mathrm{p}$. 324] to numerically evaluate the integral of $f(t)$ over $[-1,1]$ is given by

$$
E=\frac{2^{2 m+1}(m !)^{4}}{(2 m+1)[(2 m) !]^{3}} f^{(2 m)}\left(t_{0}\right), \quad\left|t_{0}\right|<1,
$$

where $f^{(2 m)}(t)$ means the $2 m$ th derivative of $f(t)$ with respect to $t$, which is assumed to exist. The integral in (33), which is the one we wish to evaluate, is transformed by

$$
u=\left[\left(\lambda-(1-x)^{1 / 2}\right) / 2\right] t+\left(\lambda+(1-x)^{1 / 2}\right) / 2,
$$

such that

$$
I_{x}(a, 1 / 2 ; \lambda)=\frac{\lambda-(1-x)^{1 / 2}}{M} \int_{-1}^{1} F(u) d t=\int_{-1}^{1} g(t) d t
$$

where

$$
F(u) \equiv\left(1-u^{2}\right)^{a-1}
$$

Similarly,

$$
\bar{I}_{x}\left(a, \frac{1}{2}\right)=\frac{(1-x)^{1 / 2}}{M} \int_{-1}^{1} F(v) d t=\int_{-1}^{1} h(t) d t .
$$

The reason $\bar{I}_{x}$ is explicitly considered here is that the total integration interval in (33) can be halved. This is done by using (39) when $\lambda / 2<(1-x)^{1 / 2}$ and by using (41) when $\lambda / 2 \geqq(1-x)^{1 / 2}$. This reduces $E^{\prime}$ by a factor of $2^{+(2 m+1)}$, which is significant. The term $\lambda / 2(\leqq 1 / 2)$ is quite small for large $a$, e.g., if $a=10^{4}$, then, from (37), $\lambda / 2=0.024$ for $\epsilon^{\prime \prime}=9 \times 10^{-11}$.

The $2 m$ th derivative of $g(t)$ and $h(t)$ is needed in (38). Letting

$$
F_{u}{ }^{(2 m)} \equiv \frac{d^{2 m} F(u)}{d u^{2 m}}
$$

one obtains

$$
\begin{array}{lll}
g^{(2 m)}\left(t_{1}\right)=\frac{2}{M}\left(\frac{\lambda-(1-x)^{1 / 2}}{2}\right)^{2 m+1} F_{u}{ }^{(2 m)}\left(u_{1}\right), & & \frac{\lambda}{2}<(1-x)^{1 / 2}, \\
h^{(2 m)}\left(t_{2}\right)=\frac{2}{M}\left(\frac{(1-x)^{1 / 2}}{2}\right)^{2 m+1} F_{u}{ }^{(2 m)}\left(v_{2}\right), & & \frac{\lambda}{2} \geqq(1-x)^{1 / 2},
\end{array}
$$

where $(1-x)^{1 / 2}<u_{1}<\lambda$ and $0<v_{2}<(1-x)^{1 / 2}$. The second factors on the right-hand sides of (43) and of (44) are bounded by $(\lambda / 4)^{2 m+1}$. The principal result we wish to derive is that

$$
E \leqq E^{\prime} \equiv \frac{2}{M}\left[\frac{(\lambda / 2)^{2 m+1}(m !)^{3}}{(2 m+1)[(2 m) !]^{2}}\right] \frac{\Gamma(a)}{\Gamma(a-m)},
$$


subject to the constraint $a-1>2 m+1 / 2$. However, since $a>60$ here, and $m$ will turn out to be about 10, the constraint is easily satisfied.

Let

$$
U_{a, r} \equiv \frac{d^{r}}{d u^{r}}\left(1-u^{2}\right)^{a}
$$

so that

$$
U_{a-1,2 m}=F_{u}{ }^{(2 m)} .
$$

It is proved in Appendix $\mathrm{B}$ of [3] that $U_{a, r}$ satisfies the following equations:

$$
\begin{aligned}
& \left(1-u^{2}\right) U_{a, r}^{\prime \prime}+2(a-r-1) u U_{a, r}^{\prime}+(2 a-r)(r+1) U_{a, r}=0 \\
& U_{a, r}=\sum_{i=0}^{\langle r / 2\rangle}(-1)^{r-1} \frac{2^{r-2} i r \Gamma(a+1)}{i !(r-2 i) ! \Gamma(a-r+i+1)} u^{r-2 i}\left(1-u^{2}\right)^{a-r+i}
\end{aligned}
$$

for nonnegative integers $r$ and real numbers $a \geqq r$.

The key fact which leads to a useful bound on $U_{a, r}$ is that the absolute values of the extrema of $U_{a, r}$ form a decreasing finite sequence on $[0,1]$ for $a>r+1 / 2$. The proof of this statement is relegated to the appendix. Assuming the statement true, there follows from (49) that

$$
\left|U_{a, 2 m}(u)\right| \leqq\left|U_{a, 2 m}(0)\right|,
$$

and

$$
\left|U_{a-1,2 m}(0)\right|=\frac{(2 m) ! \Gamma(a)}{m ! \Gamma(a-m)} .
$$

Thus (45), the desired result, is obtained by substituting (51) for (47), using this result in (43) or (44), and then (43) or (44) is used in (38). This completes the discussion on the error bound.

The explicit formulas based on the Gaussian quadrature for $I_{x}(a, 1 / 2)$ are:

$$
\begin{aligned}
& I_{x}(a, 1 / 2) \cong\left(\frac{\lambda-(1-x)^{1 / 2}}{M}\right) \\
& \times \sum_{i=1}^{m} w_{i}\left\{1-\left[(1-x)^{1 / 2}+\left(\lambda-(1-x)^{1 / 2}\right)\left(1+y_{i}\right) / 2\right]^{2}\right\}^{a-1} \\
&+\epsilon^{\prime \prime} / 2, \quad \text { if } \lambda^{2}<4(1-x), \\
& \bar{I}_{x}(a, 1 / 2) \cong\left((1-x)^{1 / 2} / M\right) \sum_{i=1}^{m} w_{i}\left[1-(1-x)\left(1+y_{i}\right)^{2} / 4\right]^{a-1}, \\
& \text { if } \lambda^{2} \geqq 4(1-x),
\end{aligned}
$$

where the $y_{i}$ and $w_{i}$ are the Gaussian abscissae and weights, respectively, of order $m$ on $[-1,1],\left[1\right.$, p. 916]. The additional factor of $\epsilon^{\prime \prime} / 2$ in (52) is accounted for by the fact that the last term in (34) is always nonnegative and no larger than $\epsilon^{\prime \prime}$. Thus for $\lambda$ satisfying (36), there follows

$$
\left|I_{x}(a, 1 / 2)-\left[I_{x}(a, 1 / 2 ; \lambda)+\epsilon^{\prime \prime} / 2\right]\right|<\epsilon^{\prime \prime} / 2 .
$$

The procedure for Case $\mathrm{C}$ has now been essentially established. One detail remains to be discussed, namely, a description of a method for computing $\ln \Gamma(s)$. 
3. Computation of $\ln \Gamma(s)$, $\ln \Gamma(a+c)-\ln \Gamma(a)$. The procedures as described above require the value of $\ln \Gamma(s)$ to high accuracy. This is achieved in the following way. If $1 / 2 \leqq s \leqq 100$, a table look-up method is used, i.e., the values of $\ln \Gamma(s)$ are stored for $s=1 / 2(1 / 2) 100$ to the full accuracy of a single-precision number (which is 14 digits on STRETCH). If $s>100$, it would seem natural to use the classical asymptotic series for $\ln \Gamma(s)$ [1, p. 267], which is given by

$$
\begin{aligned}
\ln \Gamma(s) \cong & (s-1 / 2) \ln (s-1)-(s-1)+(1 / 2) \ln 2 \pi \\
& +\frac{1}{12} \frac{1}{s-1}-\frac{1}{360} \frac{1}{(s-1)^{3}}+\frac{1}{1260} \frac{1}{(s-1)^{5}}-\cdots,
\end{aligned}
$$

where the first five terms would be enough for at least $13 \mathrm{D}$ accuracy. In every case, however, in which $\ln \Gamma(s)$ is needed, actually the difference

$$
J \equiv \ln \Gamma(a+c)-\ln \Gamma(a)
$$

occurs, where the quantity $c$ may be either $1 / 2$ or some expression such as $N-1$, $N-1 / 2$ or $N+1 / 2$. The use of (55) to compute the two logarithm terms of (56) separately leads to a prohibitive loss of significant digits if $a$ is large. This may be seen by observing that the dominant term in (55) for either $s=a+c$ or $s=a$ is of the order of $a \ln a$. Thus upon subtraction, an undesirable loss of digits occurs, e.g., if $a=10^{4}$ and $c=1 / 2$, four digits are lost. If $a=10^{8}, b=1 / 2$, then $\ln \Gamma\left(10^{8}+1 / 2\right)-\ln \Gamma\left(10^{8}\right)=1742068075.3142-1742068066.1038=9.2104$, so that in this case nine digits are lost.

This difficulty is resolved by using the following asymptotic series for $J$, when $a>100$,

$$
\begin{aligned}
\ln \Gamma(a+c)-\ln \Gamma(a) \cong & c-\frac{1}{2} \frac{c}{a}\left[\frac{\ln (1+c / a)}{c / a}-1\right] \\
& -\frac{1}{2} \frac{c}{a}+c \ln (a+c) \\
& -\frac{1}{12}\left[\frac{1}{a}-\frac{1}{a+c}\right]+\frac{1}{360}\left[\frac{1}{a^{3}}-\frac{1}{(a+c)^{3}}\right] \\
& -\frac{1}{1260}\left[\frac{1}{a^{5}}-\frac{1}{(a+c)^{5}}\right]+\cdots .
\end{aligned}
$$

This series can be derived by the use of the standard Stirling approximation (55). The first expression in square brackets on the right-hand side of (57) is evaluated by the series

$$
\frac{\ln (1+\theta)}{\theta}-1=-y+\frac{2 y^{2}}{2+\theta}\left(\frac{1}{3}+\frac{y^{2}}{5}+\frac{y^{4}}{7}+\frac{y^{6}}{9}+\cdots\right),
$$

where $\theta \equiv c / a$ and $y \equiv \theta /(2+\theta)$. The series in parentheses is efficiently generated by the obvious recurrence relation

$$
A_{n}=\left(\frac{2 n+1}{2 n+3}\right) y^{2} A_{n-1}, \quad n=1,2, \cdots,
$$

where $A_{n} \equiv y^{2 n} /(2 n+3)$ and $A_{0}=1 / 3$. Either five or ten terms of this series are used to attain $14 \mathrm{D}$ accuracy, such that if

(1) $0<\theta \leqq 0.15$ five terms are used,

(2) $0.15<\theta<0.6$ ten terms are used. 
It is also necessary to retain the series given by (55) in order to compute $\ln \Gamma(a+c)$ when its value is not stored and yet $a<100$, e.g., if $a+c=140$ and $a=90$.

4. Total Error in Computing $I_{x}(a, b)$. The computing program is set up in such a way as to compute $I_{x}(a, b)$ with an over-all error not exceeding $\epsilon$. Quantities $\epsilon^{\prime}$ and $\epsilon^{\prime \prime}$ are associated with $\epsilon$ such that $\epsilon^{\prime}=\epsilon / 4$ and $\epsilon^{\prime \prime}=\epsilon / 2$. For the evaluation of $I_{x}$ under Case C, for example, the value of $\epsilon^{\prime}$ is used to bound $E^{\prime}$ in (45) such that the truncation error due to the evaluation of $I_{x}(a, 1 / 2)$ by Gaussian quadrature does not exceed $\epsilon^{\prime}$. The value of $\epsilon^{\prime \prime}$ is used to determine $\lambda$ from (37) so that the value of $I_{1-\lambda 2}(a, 1 / 2 ; 1)$ is less than $\epsilon^{\prime \prime}$. The quantity $\epsilon^{\prime}$ is also used to terminate the summation of the $c_{i}$ in (26), if $c_{i}<\epsilon^{\prime} /(j-1)$ for some $i \geqq N$ or some $i \leqq N$. Thus the total error will not exceed $\epsilon^{\prime}+\epsilon^{\prime \prime}+\epsilon^{\prime}=\epsilon$. The details are given in [3].

The program as presently set up on STRETCH yields $I_{x}(a, b)$ to $10 \mathrm{D}$ accuracy where

$$
\epsilon=1.8 \times 10^{-10}, \quad \epsilon^{\prime}=4.5 \times 10^{-11}, \quad \epsilon^{\prime \prime}=9.0 \times 10^{-11} .
$$

The average computing time is 2.6 milliseconds; it would be about 8 milliseconds per case on an IBM 7090.

5. Appendix. This appendix contains a sketch of a proof that the absolute values of the extrema of $U_{a, r}$ as defined by (46) do not increase as a function of $u$ on $[0,1)$ provided $a>r+1 / 2$. Greater detail is given in [3].

The result is obtained with the use of the theory of ordinary differential equations $[13$, p. 99]. The theorem we employ states that if

$$
\frac{d}{d u}\left(p(u) \frac{d y}{d u}\right)+P(u) y=0
$$

such that

a. $p(u)$ and $P(u)$ and their first derivatives are continuous on $(a, b)$, i.e., $p$, $P \epsilon C^{\prime}(a, b)$,

b. $[p(u) P(u)]$ is a nondecreasing (nonincreasing) function of $u$ on $(a, b)$,

c. $P(u) \neq 0$ on $(a, b)$,

then the absolute values of the extrema, $\left|y_{m}\right|$, of any integral of $(60), y(u)$, form a nonincreasing (nondecreasing) sequence on $(a, b)$. If the hypotheses of this theorem are satisfied on the half-open interval $[a, b)$, then it is easily shown that the conclusion also holds on the half-open interval, that is, the extrema on $[a, b)$ are such that the corresponding values of $|y|$ form a nonincreasing (nondecreasing) sequence.

Eq. (48) is easily transformed to the form of (60) (see [13, p. 96]), so that (48) becomes

$$
\frac{d}{d u}\left[\left(1-u^{2}\right)^{-(a-r-1)} \frac{d y}{d u}\right]+(r+1)(2 a-r)\left(1-u^{2}\right)^{-(a-r)} y=0,
$$

where

$$
p(u) \equiv\left(1-u^{2}\right)^{-(a-r-1)}, \quad P(u) \equiv(r+1)(2 a-r)\left(1-u^{2}\right)^{-(a-r)} .
$$

Conditions a, b, c above are actually satisfied on $[0,1)$, provided $a>r+1 / 2$. 
Thus, the hypotheses of the modified theorem are satisfied, the conclusion of the modified theorem holds and contains the result which was to be proved.

\author{
U. S. Naval Weapons Laboratory \\ Dahlgren, Virginia
}

1. M. Abramowitz \& I. A. Stegun (Editors), Handbook of Mathematical Functions, National Bureau of Standards Appl. Math. Series, 55, U. S. Government Printing Office, Washington, D. C., 1964. MR 29 \#4914.

2. H. Cramér, Mathematical Methods of Statistics, Princeton Math. Series, Vol. 9, Princeton Univ. Press, Princeton, N. J., 1946. MR 8, 39.

3. A. R. DiDonato \& M. P. Jarnagin, "A method for computing the incomplete beta function ratio," NWL Report 1949 (revised), U. S. Naval Weapons Lab., Dahlgren, Virginia, 1966.

4. H. E. Fetris, "On the calculation of integrals of the form $\int_{0}^{\theta} \sin { }^{p} \phi \cos { }^{q} \phi d \phi, " J . M a t h$. Phys., v. 33, 1959, p. 283.

5. W. GaUtschi, "Incomplete beta function ratios," Comm. ACM, v. 7, 1964, p. 143.

6. F. B. Hildebrand, Introduction to Numerical Analysis, McGraw-Hill, New York, 1956. MR 17, 788.

7. O. Ludwig, "Incomplete beta ratio," Comm. $A C M$, v. 6, 1963, p. 314.

8. E. C. Molina, "Expansions for Laplacian integrals of the form $\int_{x_{1}^{2}}^{x_{2}}[y(t)]^{\theta} \Phi(t) d t$," Bell. System Tech. J., v. 11, 1932, p. 563. 1934.

9. K. Pearson, Tables of the Incomplete Beta Function, Cambridge Univ. Press, New York,

10. H. E. Soper, The Numerical Evaluation of the Incomplete B-Function or of the Integral $\int_{0}^{x} x^{p-1}(1-x)^{q-1} d x$ for Ranges of $x$ Between 0 and 1 , Tracts for Computers, No. VII, Cambridge Univ. Press, New York, 1921.

11. I. C. TANG, "On the computation of a certain type of incomplete beta functions," Comm. $A C M$, v. 6, 1963 , p. 689.

12. C. M. Thompson, "Tables of percentage points of the incomplete beta function," Biometrika, v. 32, 1941, pp. 151-181. MR 3, 153.

13. F. G. Tricomi, Differential Equations, Hafner, New York, 1961. MR 25 \#2254b.

14. M. E. WISE, "The incomplete beta function as a contour integral and a quickly converging series for its inverse," Biometrika, v. 37, 1950, pp. 208-218. MR 12, 724.

15. M. E. WISE, "The incomplete beta function and the incomplete gamma function," $J$. Roy. Statist. Soc. Ser. B, v. 10, 1948, p. 264. MR 10, 453.

16. M. E. Wise, "The use of the binomial distribution in an industrial sampling problem," Suppl. J. Roy. Statist. Soc. Ser. B, v. 8, 1946, pp .202-211. MR 9, 49.

17. J. WishaRT, "Determination of $\int_{0}^{\theta} \cos ^{n+1} \theta d \theta$ for large values of $n$, and its application to the probability integral of symmetrical frequency curves," Biometrika, v. 17, 1925, pp. 68, 469.

18. Staff of the Computation Laboratory, Tables of the Cumulative Binomial Probability Distribution, Harvard Univ. Press, Cambridge, Mass., 1955. MR 18, 517.

19. National Bureau of Standards, Tables of the Binomial Probability Distribution, Appl. Math. Series, No. 6, U. S. Government Printing Office, Washington, D. C., 1950. 